\title{
Immunolocalization of the Glioblastoma Cells by Nanoparticles Using Microscopy Tools
}

\author{
Lorena Favaro Pavon et al. ${ }^{*}$ \\ ${ }^{1}$ Instituto do Cérebro - Instituto Israelita de Ensino \\ e Pesquisa Albert Einstein - IIEPAE, São Paulo \\ Brasil
}

\section{Introduction}

According to World Health Organization (WHO 2007), tumors of the nervous system are classified into seven major groups including primary tumors (neuroepithelial, meningeal, cranial nerves, paraspinal nerves, germinal, region sellar and hematopoietic) and the secondary tumors or metastatic.

The primary tumors of the nervous systems are relatively rare. According to data from the Central Tumor Registry of the Central Nervous System from Unites States (CBTRUS 2011), it is estimates that about 64.530 new cases (approximately 22.020 of them malignant) diagnosed in 2010, representing $1.44 \%$ of all malignancies diagnosed in this country. Despite the low incidence, malignant primary tumors are highly lethal - responsible for about 13.140 deaths in the Unites States in 2010.

Gliomas are tumors that arise from glial cells and include astrocytomas, oligodendrogliomas, oligoastrocitomas (also called mixed gliomas) and ependymomas. Gliomas are among the group of neuroepithelial tumors and account for $31 \%$ of primary tumors and $80 \%$ of malignant tumors of the nervous system. The astrocytoma group represents $76 \%$ of all gliomas, and glioblastoma represents $53.7 \%$ of cases (CBTRUS 2011). Glioblastoma is the most frequent and malignant astrocytoma, and despite numerous advances in the diagnosis and treatment of these tumors, their prognosis remains limited (Brandes et al. 2008; Wen; Kesari, 2008).

\footnotetext{
${ }^{*}$ Luciana Cavalheiro Marti², Tatiana Tais Sibov' ${ }^{1}$, Suzana M.F. Malheiros ${ }^{3}$, Daniela Mara Oliveira ${ }^{4}$, Maria Izabel Camargo-Mathias ${ }^{5}$, Edson Amaro Junior ${ }^{1,6}$, Reynaldo Andre Brandt ${ }^{7}$, Jorge Roberto Pagura ${ }^{7}$, Marcos Augusto Stavale ${ }^{7}$, Guilherme Carvalhal Ribas ${ }^{7}$ and Lionel Fernel Gamarra ${ }^{1}$

${ }^{1}$ Instituto do Cérebro - Instituto Israelita de Ensino e Pesquisa Albert Einstein - IIEPAE, São Paulo,

${ }^{2}$ Centro de Pesquisa Experimental - Instituto Israelita de Ensino e Pesquisa Albert Einstein - IIEPAE, São Paulo,

${ }^{3}$ Departamento de Neurologia e Neurocirurgia - Universidade Federal de São Paulo - UNIFESP, São Paulo,

${ }^{4}$ Universidade de Brasília, Brasília, DF

${ }^{5}$ Departamento de Biologia, Instituto de Biociências - Universidade Estadual Paulista - UNESP, Rio Claro, SP

${ }^{6}$ Departamento de Radiologia, Universidade de São Paulo - USP, São Paulo,

${ }^{7}$ Programa Integrado Neuro-Oncologia, Hospital Albert Einstein, São Paulo, SP

Brasil
} 
It is described that the glioma is the most common neoplasm of the human central nervous system (CBTRUS 2011), being difficult therapeutic approach, due to several factors: i) presenting infiltrative ii) possess tumor composed of cells with different morphofunctional characteristics that express a variety of neural markers, iii) be highly resistant to radiotherapy and chemotherapy procedures (Singh et al., 2003).

Glioblastoma cell lineages express a set of cell surface antigens also found in mesenchymal stem cells (MSC), such as CD29, CD44, CD73 (SH3, SH4), CD90 (Thy-1) and CD105 (SH2) (Sordi et al., 2005). In addition, a small population of cancer stem cells has been identified in brain (Singh et al., 2004). Some evidence suggests that CD133 is a marker for a subset of glioblastoma cancer stem cells. The MSC-like phenotype and CD133 positive cells isolated from human glioblastoma may play a role in tumorigenesis or tumor recurrence and could be a potential target for therapies for glioblastoma. The transcription factor Forkhead BoxM1 (FoxM1) also has been described as up-regulated in glioblastoma cells and may act as a malignancy marker.

Nanobiotechnologic technologies can offer excellent tools for evaluating and understanding tumorigenesis, tumor mapping and tumor behaviour. One example is the use of superparamagnetic iron oxide nanoparticles (SPIONS). Recently, SPIONs have attracted attention for their use in nanotechnology (Tartaj et al., 2003) and biomedical applications, including drug delivery (Voltairas et al., 2002), hyperthermia in cancer treatment (Jordan et al., 1997; Jordan et al., 1999) and as image contrast agents in magnetic resonance imaging (MRI) (Cheng et al., 2005; Kumar et al., 2007).

It was also used in this study, superparamagnetics iron oxide nanoparticles for cell separation process with MACS Technology. Applying the magnetic cell sorting (MACS) technology, we reported results of purifying the CD133 positive cells from the glioblastoma cells. In malignant brain tumors, CD133 has been suggested to be a cancer stem cell marker, since only CD133 positive cells from brain tumor biopsy material were able to initiate brain cancer in a mouse model (Singh et al., 2004). Prominin-1 (PROM-1), also called CD133, is a protein with several isoforms of unknown physiological or pathological function, and is localized both in the cytoplasm and at the cell surface (Miraglia, et al. 1997). It is expressed by human neural stem cells and has been proposed to have a function in central nervous system development.

Another possible nanobiotechnologic tool of great relevance are the Quantum Dots (QD). Semiconductor QDs are nanoparticles that have attracted widespread interest in biology and medicine due to their unique optical and electronic properties. These properties, especially their reduced tendency to photobleach and the dependence of their fluorescence wavelength on their size, make them suitable for fluorescent probing applications to detect cancer biomarkers in vitro and in vivo in cells. Thus, the QD act as probes which are detectable by the optical techniques of spectroscopy and magnetic resonance imaging (Lee et al., 2007) including the technique of transmission electronic microscopy (TEM), due to the nanoparticles' electrondensity (Nisman et al., 2004).

The present study analyzed the use of nanoparticles for labelling human glioblastoma cell lineages (U87MG/A172). For this purpose, we used SPIONs, as well as monoclonal antibodies (CD29, CD44, CD105) or FOXM1 conjoined to secondary antibodies linked to quantum dots. This strategy was based on complete glioblastoma immunophenotypic profile determined by flow cytometry. Then, the analysis was performed by transmission electron microscopy and immunofluorescence glioblastoma cells using superparamagnetic 
nanoparticles, SPIONs and QD. We also described the immunolocalization of CD133 epitopes in cells from primary cultures derived from human glioblastoma.

\section{Material and methods}

\subsection{Culture and labelling human glioblastoma lineages with quantum dots (QD)}

The cell lines of human glioblastoma (U87MG and A172) were maintained in a culture of DMEM-LG: Dulbeccos Modified Eagle Medium Low Glucose to 10\% Fetal Bovine Serum and 1\% Antibiotic-Antimycotic (100X) and 200nM L-Glutamine (100X).

Human glioblastoma cells were labeled in the culture after their expansion and adhesion to the culture plate according to the manufacturer's instructions (Invitrogen). Briefly, cells were incubated with primary antibodies, such as CD29, CD44 and CD105 followed by incubation with secondary antibodies (pure goat anti-mouse, IgG) linked to quantum dots (QD 565nm). The lineages were labeled for the transcription factor FOXM1 (Zimed - Invitrogen), which was conjoined in advance with quantum dots (QD 525nm) according to the directions of the QD Antibody Conjugate Kit (Invitrogen).

The labeled glioblastoma cells were maintained in a humidified incubator (Thermo Fisher Scientific Inc. 3110, Waltham, MA) with $5 \% \mathrm{CO}_{2}$ at $37^{\circ} \mathrm{C}$ for 24 hours and subsequently fixed in $1 \%$ glutaraldehyde for later analysis in TEM (Philips CM100).

\subsection{SPIONs synthesis and glioblastoma lineages labelling}

The colloidal suspensions based on SPIONs $\left(\mathrm{Fe}_{3} \mathrm{O}_{4}\right)$ were previously synthesized by the solgel process. These syntheses included the rapid hydrolysis of $\mathrm{Fe}^{3+}$, by the addition of ammonium hydroxide to the $0.2 \mathrm{M} \mathrm{FeCl}_{3} \mathrm{H}_{2} \mathrm{O}$ aqueous solution in the presence of a surfactant (dextran). The dialysis of the precipitate permitted its peptizing leading to the formation of a colloidal suspension with SPIONs which have a diameter of $6 \mathrm{~nm}$.

Thus, the tumor cells lineages were labeled in the culture with a concentration of $0.5 \%$ SPIONs and kept in a humidified incubator (Thermo Fisher Scientific Inc. 3110, Waltham, MA) with $5 \% \mathrm{CO}_{2}$ at $37^{\circ} \mathrm{C}$ for 24 hours. Subsequently, they were fixed in $4 \%$ paraformaldehyde and stained with 5\% Prussian blue, viewed under a light microscope (OLYMPUS IX51), and fixed in 1\% glutaraldehyde for later analysis under a TEM (Philips CM100).

\subsection{The immunophenotyping of glioblastoma lineage cells by flow cytometry}

The established glioblastoma lineage cells were analyzed for the cell-surface expression of typical mesenchymal stem cell protein markers. These experiments were conducted using monoclonal antibodies commercially available. The staining technique followed the manufacturer's instructions. Briefly, the samples in the $4^{\text {th }}$ stage were harvested by a treatment with $0.25 \%$ Tryple Express (GIBCO-Invitrogen, Carlsbad, CA), washed with PBS $(\mathrm{pH}=7.4)$ and stained with the selected monoclonal antibodies and incubated in the dark for 30 minutes at $4{ }^{\circ} \mathrm{C}$. Cells were then washed and fixed with $1 \%$ paraformaldehyde. The following human antibodies were used: CD14-FITC (clone: M5E2; BD Pharmingen - San Diego - CA), CD29-PE (clone: MAR4; BD Pharmingen - San Diego - CA), CD31-PE (clone: WM59; BD Pharmingen - San Diego - CA), CD34-PE (clone: 581; BD Pharmingen - San Diego - CA), CD44-PE (clone: 515; BD Pharmingen - San Diego - CA), CD45-PerCP-Cy5 (clone: 2D1; Biosciences - San Jose - CA), CD73-PE (clone: AD2; BD Pharmingen - San Diego - CA), CD90-APC (clone: 5E10; BD Pharmingen - San Diego - CA), CD106-FITC 
(clone: 51-10C9; BD Pharmingen - San Diego - CA), CD166-PE (clone: 3A6; BD Pharmingen - San Diego - CA), HLA-DR-PerCP-Cy5 (clone: L243; Biosciences - San Jose - CA) and CD105-PE (clone: 8E11; Chemicon - Temecula - CA).

The data acquisition was carried out by FACSARIA flow cytometry equipment (BD Biosciences, San Jose, CA) and data analyses were performed using FACSDIVA software (BD Biosciences, San Jose, CA) or Flow Jo Software (TreeStar, Ashland, OR).

\subsection{Culture of primary glioblastoma cells}

Glioblastoma tumor cell samples were washed with 1\%PBS and the enzymatic dissociation was performed in $0.3 \%$ collagenase. The obtained cells were them resuspended in

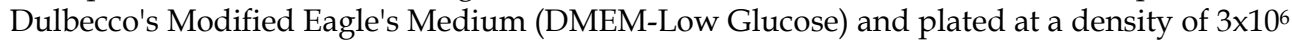
live cells/60mm plate.

\subsection{Magnetic cell separation using MACS (Miltenyi Biotec)}

The culture of primary glioblastoma cells were purified following the separation protocol by MiniMACS microbeads affinity chromatography using anti-CD133 bound to magnetic beads (Miltenyi Biotec).

The cells were filtrated in $30 \mu \mathrm{m}$ nylon filters and the cell number was determined by an automatic cell counter (Coulter). Afterwards, the cells were centrifuged (400g for 5 minutes) and resuspended $300 \mu \mathrm{L}$ of PBS solution containing $2 \mathrm{mM}$ EDTA and $0.5 \%$ BSA (solution 1 ) for each $10^{8}$ cells. The next step was the addition of $100 \mu \mathrm{L}$ of FcR blocker and $100 \mu \mathrm{L}$ of magnetic micro-spheres with $\mathrm{CD} 133^{+}$antibodies, followed by the incubation at $6^{\circ} \mathrm{C}$ for 30 minutes.

After incubation, the labelled cells were centrifuged and resuspended in solution 1 to be separated in the chromatography column to isolate only the CD133+ cells. The selected CD133+ cells were subsequently fixed in $1 \%$ glutaraldehyde for later analysis in a TEM.

\subsection{Immunocytochemical staining of primary culture glioblastoma}

Glioblastoma tumor samples were washed 1\% PBS and tumor cells were disaggregated in a solution containing $0.3 \%$ collagenase in $1 \%$ PBS buffer. Cells derived from the tumor were then resuspended in Dulbecco's Modified Eagle's Medium (DMEM -Low Glucose) and plated at a density of $3 \times 10^{6}$ live cells/60mm plate. Cells were fixed with $4 \%$ paraformaldehyde and stained with antibody against CD133/1 (human monoclonal IgG1; 1:1000 dilution; Miltenyi Biotec). After washing, the cells were incubated with KIT Advanced TMHRP Dako (K4067) Advanced TMHRP Enzyme and followed by the application of the substrate-chromogen solution $\left(\mathrm{DAB}^{+}\right)$.

\subsection{QD labelled primary culture glioblastoma by fluorescence microscopy:}

Primary antibodies CD29 in conjunction with the secondary (anti-mouse IgG) linked to 565nm nanocrystals QD (Qtracker 565 Cell Labelling Kit, Invitrogen, Carlsbad, CA) were used to label of the primary culture glioblastoma according to the manufactory's instruction. The labelling of glioblastoma cells was detected by using a fluorescence microscopy (IX51 Olympus) with a filter for detection of the QDs (QD655-A-OMF-ZERO filter, Semrock, Rochester, NY).

\subsection{Transmission electron microscopy:}

Glioblastoma lineages $\left(10^{7}\right.$ cells $)$ were fixed in $1 \%$ glutaraldehyde and $0.2 \mathrm{M}$ cacodylate buffer for two hours at $4^{\circ} \mathrm{C}$. Cells were washed in cacodylate buffer, 2 times for 15 minutes 
each. Post-fixation was performed in $1 \%$ osmium tetroxide for one hour at $4^{\circ} \mathrm{C}$, followed by another two 15 minute washes in the same buffer. For contrast, the pellet was immersed in a solution of uranyl acetate in acetone for 30 minutes. After dehydration, the material was embedded in Epon resin diluted in acetone (1:1) and incubated at $4^{\circ} \mathrm{C}$ with agitation for 24 hours. The pellet was then transferred to pure Epon resin and incubated at $60^{\circ} \mathrm{C}$ for 72 hours, until completely polymerized. Semi and ultrathin sections were obtained with the aid of a Porter Blum ultramicrotome. The semithin sections were stained with azur II (1\%) and methylene blue $(1 \%)$. The ultrathin sections were placed on copper grids and stained with uranyl acetate and lead citrate. The grids were studied and photographed under a TEM (PHILIPS CM100).

\section{Results}

\subsection{The immunophenotypic profile of glioblastoma lineage cells:}

The glioblastoma lineage cells were analyzed by flow cytometry and gated according their granularity versus size, and then followed by the surface markers analysis. These cells were analyzed for the expression of cell membrane proteins and found to be positive for the expressions of CD29, CD44, CD73, CD90, CD105, which are generally considered as a marker set for mesenchymal stem cells (Figure 1).

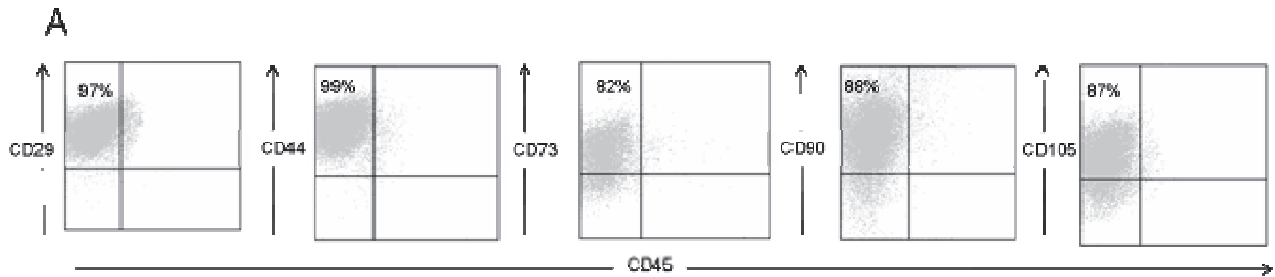

B

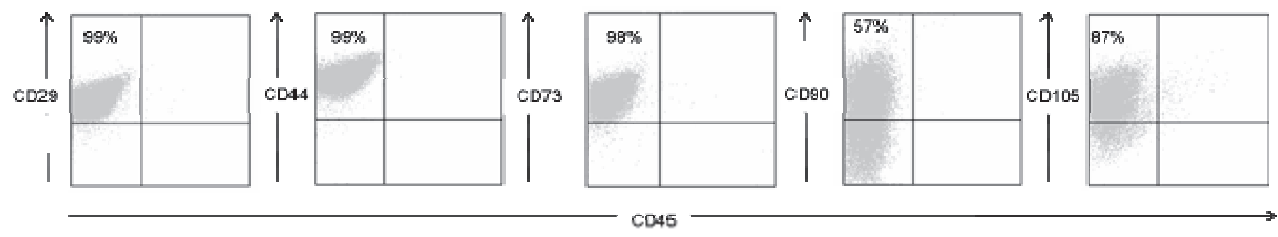

Fig. 1. Expression of mesenchymal markers as CD29, CD44, CD73, CD90 and CD105 on the cell of glioblastoma lineage A172 (A). Besides a variation on the percentage of CD73 and $\mathrm{CD} 90$, the same markers were found on the glioblastoma lineage U87MG (B).

\subsection{Glioblastoma labelling by using monoclonal antibodies such as CD29, CD44, CD105 and the transcription factor FOxM1 conjugated to quantum dots (QD $565 \mathrm{~nm}$ and $525 \mathrm{~nm}$ )}

The analysis of ultrastructural immunolocalization revealed the presence of electrondense clusters on the surface of tumor cells (Figure 2B), as well as scattered in the cytoplasm (Figures 2D; 4B, C) and in the interior of the vesicles (Figures 2E; 4B, D, F). This electrondensity demonstrates the presence of monoclonal antibodies linked to QD. 

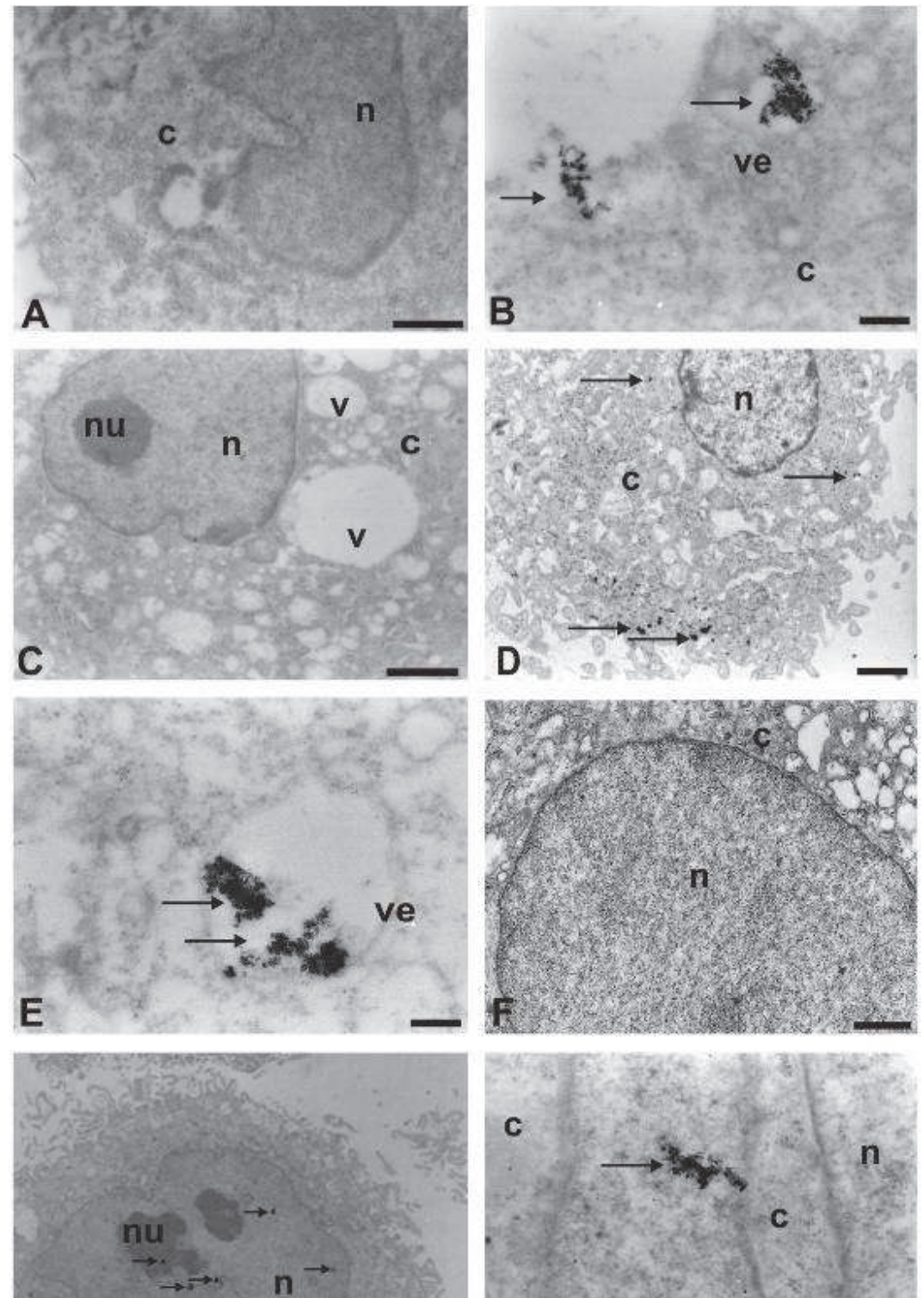

Fig. 2. Ultrastructural analysis of glioblastoma lineages marked with nanocrystal quantum dots (QDs) using TEM. B) QDs (565nm) conjoined with the CD29 monoclonal antibody. D, E) QDs (565nm) conjoined with the CD44 monoclonal antibody. G, H) QDs (525nm) conjoined with the FOxM1 transcription factor. A, C, F) Glioblastoma lineage cells of the control group. A, B, C, H) Scale: $0.25 \mu \mathrm{m}$. D, E, G) Scale: $0.5 \mu \mathrm{m}$. F) Scale: $2 \mu \mathrm{m} . \mathrm{n}=$ nucleus; c = cytoplasm; $\mathrm{nu}=$ nucleolus; $\mathrm{v}=$ vacuole; $\mathrm{ve}=$ vesicle; arrow $=$ electrondense nanoparticles (nanocrystals). 
Figure $4 \mathrm{~B}$ shows the presence of vesicles from the process of invagination of plasmic membrane.

Electrondense clusters were not observed in our control group U87MG and A172 lineage cells, which did not receive the QDs (Figures 2A, C, F; 4A). These QDs can also be associated with cytoplasmic organelles such as the rough endoplasmic reticulum (Figure 4E).

The transcription factor FOxM1 can also be evidenced by the QD electrondensity conjoined to this nuclear transcription factor. This compound can be observed by granules in various regions of the tumor cell nucleus (Figures $2 \mathrm{G}, \mathrm{H}$ ).

The figures $3 \mathrm{~A}, \mathrm{~B}$ e $\mathrm{C}$ shows, by fluorescence microscopy, the glioblastoma cell membrane staining by both CD29 primary antibody and the secondary anti-mouse antibody and, as a control, the glioblastoma cells were stained with the secondary anti-mouse antibody only (Figure 3D). The analysis of ultrastructural revealed the presence of electrondense clusters on the surface of tumor cells (Figure 3E) staining by of the primary antibody CD29 and secondary anti-mouse polyclonal antibody. Electrondense clusters were not observed in control group (Figure 3F), depicts the glioblastoma cells stained with the secondary anti-mouse antibody only, showing no unspecific staining by this antibody in these experiments.

\subsection{Primary culture glioblastoma marked with superparamagnetic nanoparticles using anti-CD133 bound to magnetic beads}

The analysis of immunocytochemistry demonstrated CD133 expression in primary culture glioblastoma (Figure 5B). Under the electron microscopy, ultrastructural analysis highlighted the presence of electrondense granules in the glioblastoma cell surface. This demonstrates the presence of anti-CD133 monoclonal antibodies bound to superparamagnetic nanoparticles recognizing the CD133 membrane protein (Figures 5D, E). This does not occur in the cells of the control group (CD133-) (Figure 5C). Electrondense signals related to superparamagnetic nanoparticles were also observed in the cell cytoplasm, suggesting their internalization through the process of the endocytosis of QD bound to the antibodies (Figures 5D, E). CD133+ cells incorporated superparamagnetic nanoparticles through its small cytoplasmic projections forming the pinocytics vesicles, as shown in the figures $5 \mathrm{E}$ and $5 \mathrm{~F}$.

\subsection{The glioblastoma lineage iron oxide superparamagnetic nanoparticles (SPIONs) markings}

The analysis of light microscopy, with Prussian blue staining, revealed large amounts of iron oxide nanoparticles dispersed in the cytoplasm of tumor cells (Figure 6B), compared to cells from the control group (Figure 6A).

The ultrastructural SPIONs markings, using electrondensity resources, confirmed the presence of these nanoparticles, mainly in the interior of the vesicles (Figures 6D, E, F, G), that did not occur in the control group cells (Figure 6C). Figure 4D showed the process of internalization of the nanoparticles, as well as invaginations of the plasma membrane and subsequent vesicle formation.

Some cells showed intense cytoplasmic vacuolation (Figure 6G), loss of cell boundaries or disintegration of the plasmic membrane (Figure $6 \mathrm{~F}$ ), and the formation of concentric lamellae forming myelin figures (Figure 6H). 

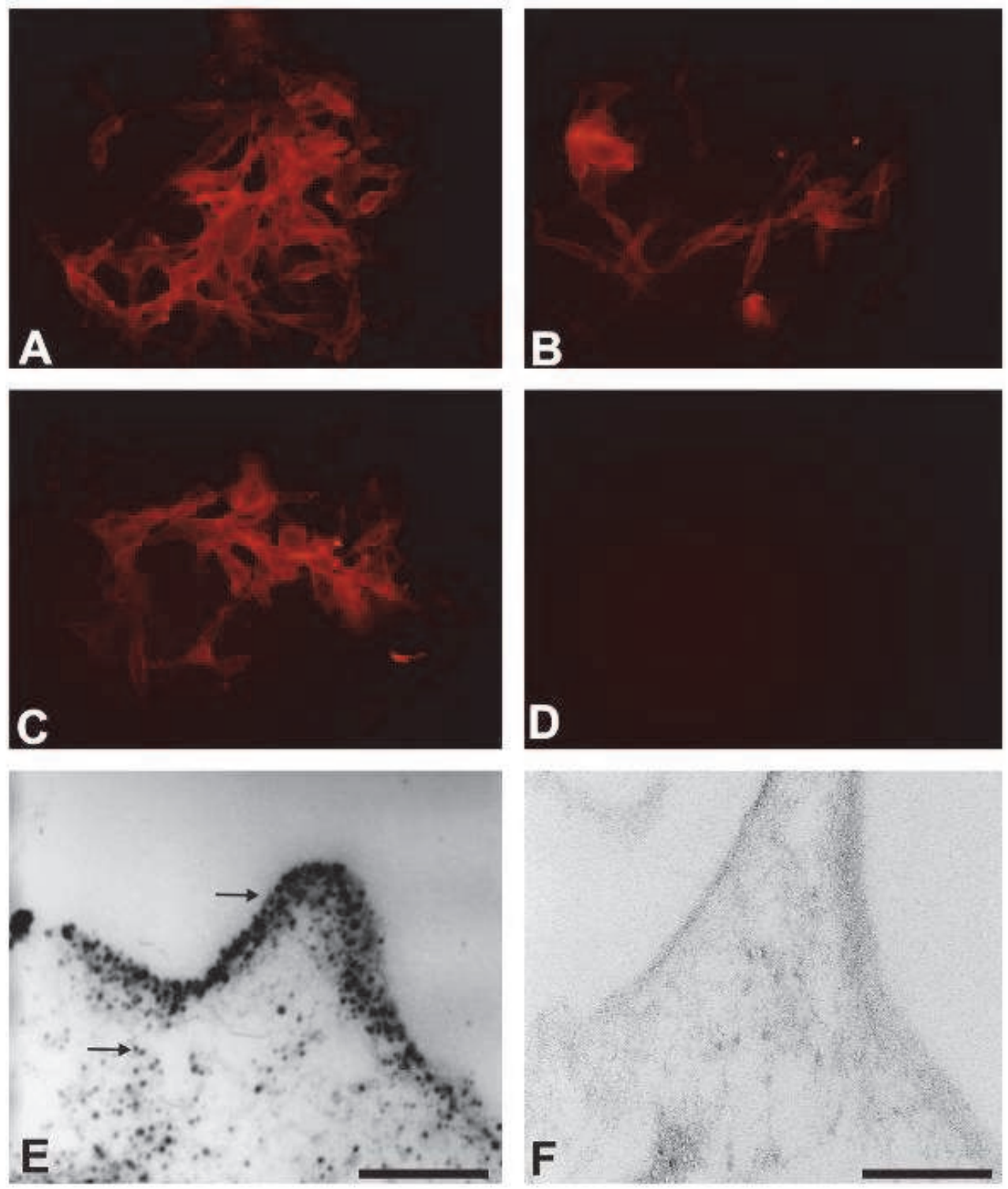

Fig. 3. Immunofluorescence and ultrastructural analysis of glioblastoma lineages marked with nanocrystal quantum dots (QDs). A, B, C, E) 565nm QDs conjoined with the CD44 monoclonal antibody. D, F) Glioblastoma cells with the secondary anti-mouse only. A, B, C, D) 600X. E, F) Scale: $0.25 \mu \mathrm{m}$. 

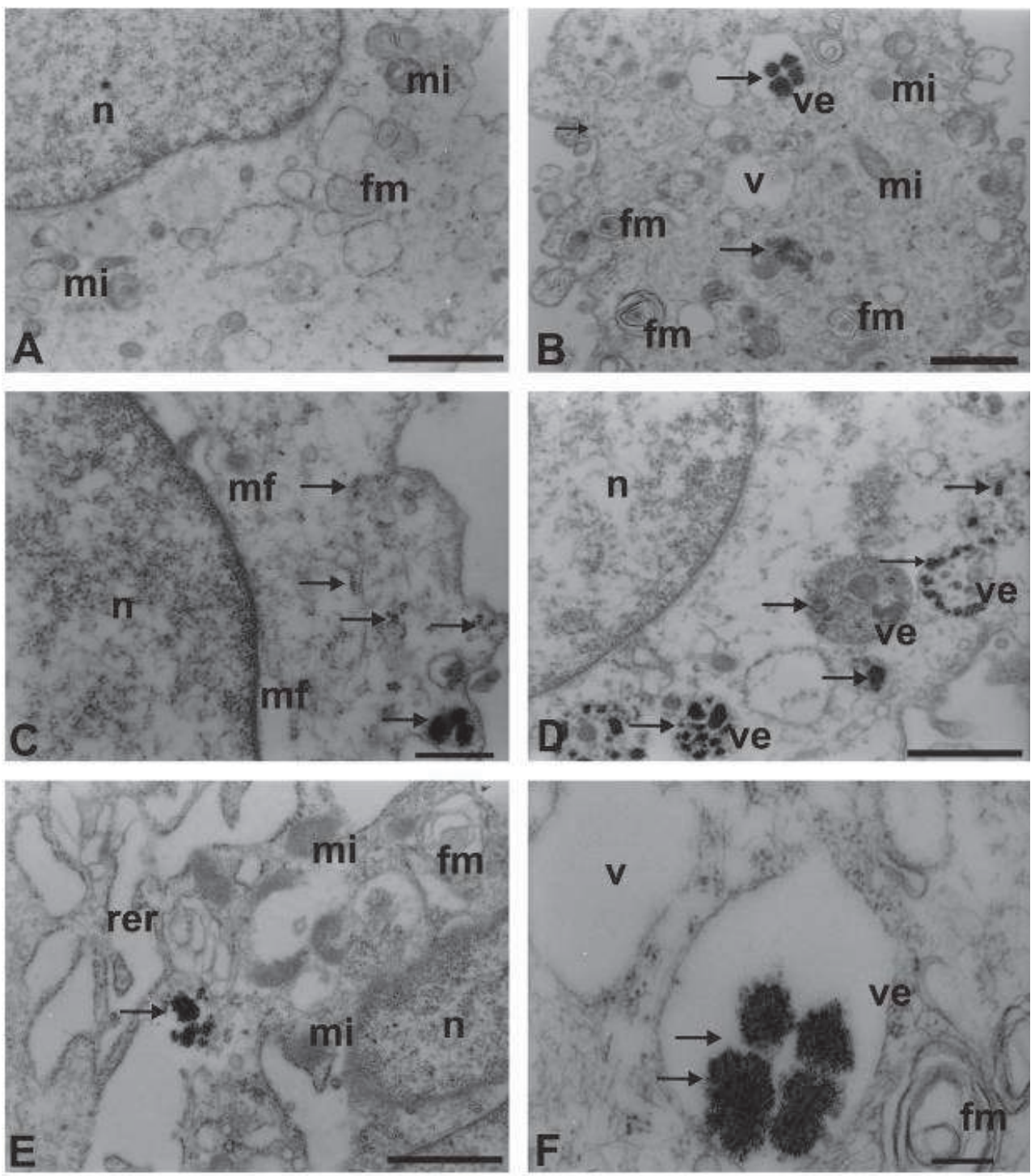

Fig. 4. B-F) Ultrastructural analysis of glioblastoma lineages marked with nanocrystal quantum dots (QD 565nm) conjoined with the CD105 monoclonal antibody. A) Glioblastoma lineage cells of the control group. A, C, D, E, F) Scale: $0.5 \mu \mathrm{m}$. B) Scale: $1 \mu \mathrm{m} . \mathrm{n}=$ nucleus; $\mathrm{v}=$ vacuole; $\mathrm{mi}=$ mitochondria; $\mathrm{ve}=$ vesicle $\mathrm{mf}=$ microfilaments; $\mathrm{fm}=$ myelin figure; rer = rough endoplasmic reticule; arrow = electrondense nanoparticles (nanocrystals). 

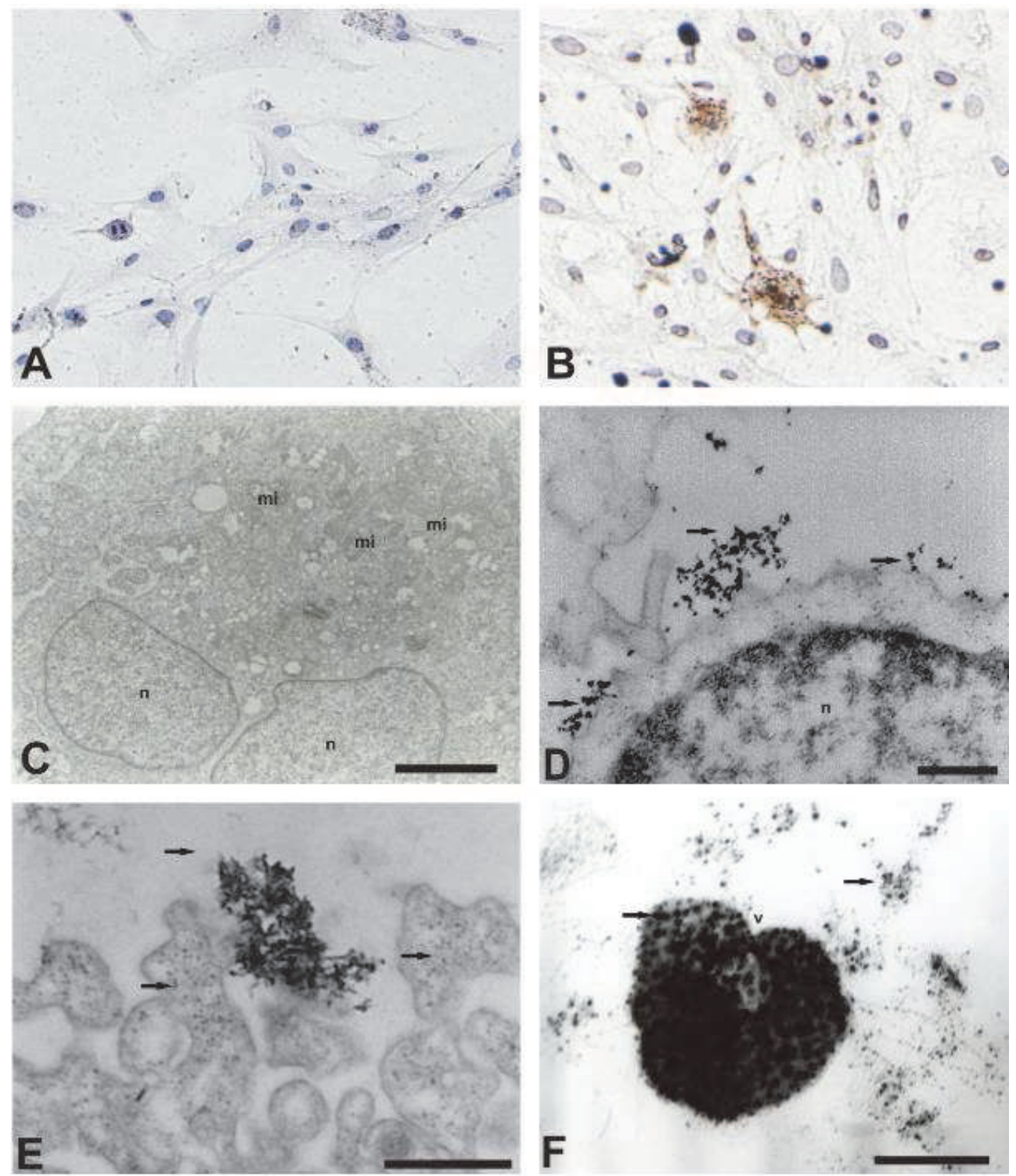

Fig. 5. Analysis of primary culture glioblastoma marked with microbeads CD133 by using immunocytochemical staining and transmission electron microscopy. (A, C) Primary culture glioblastoma of the control group. (B, D-F) Glioblastoma cells (CD133+). (A, B) 400. Scale bars: (C) $0.25 \mu \mathrm{m}$; (D, E) $0.5 \mu \mathrm{m}$; (F) $1 \mu \mathrm{m}$. n, nucleus; c, cytoplasm; mi, mitochondria. Arrows indicate electrondense nanoparticles. 

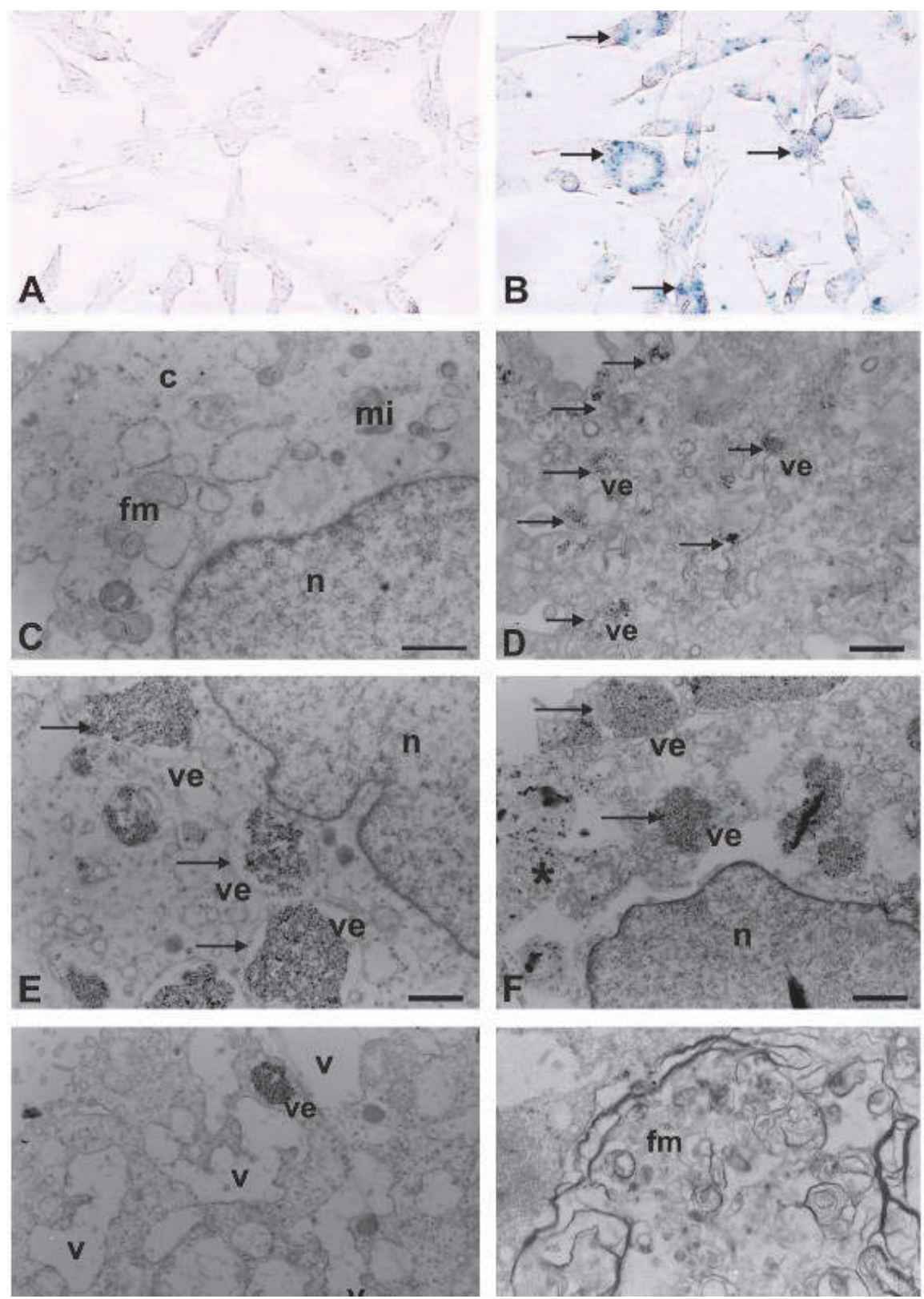

Fig. 6. Glioblastoma lineages marked with SPIONs. A, B) Light microscopy using Prussian blue staining. A, C) Glioblastoma lineage cells of the control group. A, B) 600X. C-H) Transmission electron microscopy. $\mathrm{C}-\mathrm{G}$ ) Scale: $1 \mu \mathrm{m} ; \mathrm{H}$ ) Scale: $0.25 \mu \mathrm{m} . \mathrm{n}=$ nucleus; $\mathrm{v}=$ vacuole; ve = vesicle; $\mathrm{fm}=$ myelin figure; $\mathrm{mi}=$ mitochondria $;{ }^{*}=$ cell margin; arrow $=$ electrondense nanoparticles. 


\section{Discussion}

Glioblastoma is the most malignant brain tumor (Louis et al., 2007). Because of its invasive nature, it cannot be completely removed, reducing the success of chemo and/or radiotherapy procedures (Louis et al., 2007).

Some nanobiotechnology resources, using nanoparticles in the process of cell labelling, can be extended to areas of neuro-oncology trough the study of tumorigenic cells in the immunolocalization process (Simberg et al., 2007).

Our study suggests new approaches for the identification of the glioblastoma cells by ultrastructural examination using nanobiomarkers and it can contribute for further in vivo studies by understanding glioblastoma staining process.

Immunophenotypically, the glioblastoma lineages express a set of cell surface antigens also found in mesenchymal stem cells (MSC), such as CD29 ( $\beta 1$ integrin - receptor protein of extracellular matrix and a cell to cell interaction mediator), CD44 (hyaluronate receptor), CD73 (SH3, SH4), CD90 (Thy-1) and CD105 (SH2) (Sordi et al., 2005). These finding suggest that a subset of primary glioblastoma derives from transformed cells containing MSC-like properties with partial phenotypic aspects of the MSC.

Alternatively, glioblastomas cells could activate a series of pathways that result in the establishment of the cancer cells mesenchymal phenotype which may contributes to the tumor growth and malignant progression (Tso et al., 2006). Thus, MSC-like phenotype may play a role in tumorigenesis, invasive progression or tumor recurrence.

The analysis of ultrastructural immunolocalization of glioblastoma lineages showed the presence of monoclonal antibodies CD29, CD44 and CD105 linked to quantum dots, i.e., electrondense clusters on the surface of tumor cells and in vesicles.

The electronic micrographs described an important ultrastructural finding related to the presence of vesicles that internalize QD by the process of pinocytosis for all antibodies tested. We suggest that in our study the pynocytisis occur by receptor-mediated endocytosis. The endocytosis process happens after the antibody biding to the receptor, and then a depression arises on the cell membrane which is followed by the formation of the pinocytic vesicles. These pinocytic vesicles that are surrounded by the cytoplasmic proteins called clatrines are internalized.

Immunolocalization of the process by marking cells with CD29 primary antibody described control parameters for the study. This figure 6 shows, by microscopy fluorescence, the membrane staining by both CD29 primary antibody and the secondary anti-mouse antibody and, as a control, the cells were stained with the secondary anti-mouse antibody only. These results show that there is no unspecific reaction by the anti-mouse secondary polyclonal antibody in this experiment. The electronic micrographs also described the cells stained with the primary antibody CD29 and secondary anti-mouse polyclonal antibody, which the cells stained with the secondary anti-mouse antibody only, showing no unspecific staining by this antibody in these experiments.

The CD44 monoclonal antibody selected for this study is a transmembrane glycoprotein which acts primarily as a recipient of hyaluronana, and can bind to certain other ligands in an extracellular matrix. A correlation between the particular pattern of CD44 variants produced by strains of glioblastoma cells and tumor clinicopathological parameters, such as cell motility, invasive properties, potential for metastasis, and tumor cells survival could be established (Rudzki et al., 1997). 
The CD105 monoclonal antibody, also included in this study, is intensely expressed in the tumor vascular system and can be an important indicator of prognosis of glioblastoma malignancy (Margaritescu et al., 2008).

Tumor growth and metastasis depend on the formation of new blood vessels. Tumor cells release a variety of compounds, which can act on endothelial progenitor cells activation, proliferation, and migration promoting neovascularization and consequent tumor angiogenesis (Reddy et al., 2006). Our study suggests the use of QD for tumor accumulation mechanism studies as well as targets for vascular mapping and consequent removal of tumorigenic glioblastoma cells.

Our study also proposes the use of a transcription factor called Forkhead Box M1 (FoxM1) previously conjoined with QD, as a glioblastoma marker, since this factor is described as upregulated in glioblastoma cells (Liu et al., 2006). FoxM1 was ultrastructurally evident by the nanocrystal electrondensity conjoined to this nuclear transcription factor.

Reports showed that the level of protein expression in human gliomas tissues was directly related to the tumor grade or the glioblastoma cells tumorigenicity and may act as an index of tumor malignancy.

The electronic micrographs also demonstrated that glioblastoma cell lineages internalize the $\mathrm{QD}$, expressing them ultrastructurally as electrondense structures that are located in different cellular regions, such as the cytoplasm or the rough endoplasmic reticulum.

The same process of tumor QD-marking was described by Jackson, et al. (2007) where the nanocrystals were phagocytised by macrophages and microglia, thus infiltrating experimental gliomas. Therefore, his study suggests the use of non-conjoined nanocrystals to assist the imaging system for surgical termination or glioblastoma tumor biopsies.

The analysis of immunocytochemistry demonstrated the CD133 expression in primary culture glioblastoma. Ultrastructural results showed anti-CD133 bound to the SPIONs by the presence of electrondense granules in cell membrane, as well as in the cytoplasm or forming vesicles.

CD133 localization in membrane protrusions suggests an involvement in the dynamic organization of membrane and therefore in the mechanisms influencing cell polarity, migration and interaction of stem cells with neighboring cells and/or extracellular matrix, but experimental data are currently lacking. In addition, it is not known whether CD133 has a role in self-renewal and differentiation of stem cells, witch has important implication in gliomagenesis (Singh et al., 2003; Beier et al., 2007; Zeppernick et al., 2008).

CD133 positive cells isolated from human brain tumours exhibit stem cells properties in vitro (Singh et al., 2003) and are able to initiate and drive tumor progression in vivo (Singh et al., 2004) strongly suggesting that CD133 positive cells might be the brain tumor initiating cells. This notion has been recently challenged by studies demonstrating that glioblastoma CD133 negative cells have also properties of stem cells and are tumorigenic when engrafted intracerebrally into nude mice (Beier et al., 2007).

Zeppernick, et al. (2008), for the first time, presented a direct link between the expression of a cancer stem cell antigen CD133 and patient survival in gliomas, giving support to the current cancer stem cell hypothesis. The authors demonstrated that the proportion of CD133 positive cells was an independent risk factor for tumor regrowth and time course of malignant progression in WHO grade 2 and 3 tumors. These data provide strong supportive evidence for image mapping the cancer stem cell structural model and the clinical relevance of the CD133 positive cell population in glioblastoma. 
Iron oxide superparamagnetic nanoparticles (SPIONs) were also targets in the marking of glioblastoma cells. The SPIONs were enclosed by tumor cells through endocytosis and revealed by the Prussian blue cytochemical staining or the ultrastructural electrondensity resource within the vesicles.

Our work suggests the use of SPIONs as a contrast agent in magnetic resonance imaging techniques through the internalization of iron oxide by the endocytosis process (fluid phase - mediated receptor for transferrin linked to the oxide iron) (Daldrup-Link et al., 2003).

Transmission electron microscopy studies, using iron oxide-based markers, revealed no significant morphostructural changes in the tumor marked cells (Zeppernick et al., 2008). The results for the glioblastoma lineage markings by SPIONs describe, however, intense cytoplasmic vacuolation, loss of cell boundaries or disintegration of the plasma membrane and the formation of concentric lamellae forming myelin figures. Such indicators suggest ultrastructural signs of cell death probably derived from the process of marking, corroborating findings in the Alzheimer's disease studies using magnetic nanospheres (Hartig et al., 2003).

The ultrastructural findings of the tumorigenic glioblastoma cell labelling process using quantum dot and SPIONs suggest that these nanobiomarkers can play important role in cancer diagnosis and also help to understand tumor behavior, i.e., biomimetic amplification of tumoral "homing" revealing tumor invasive properties and its potential for metastasis, recognizing vascular targets for image mapping and treatment of brain tumors (Simberg et al., 2007), and describing the tumorigenicity of glioblastoma through therapeutic indicators of tumor malignancy (Voura et al., 2004; Simberg et al., 2007),

The process of tumor cell labelling in vitro by nanoparticles, using microscopic analysis, can successfully contribute in the future to the identification of the tumorigenic cells and consequently for better understanding glioblastoma genesis and recurrence. In addition, this method may help further studies in tumor imaging, prognostic markers detection, as well as to understand the selection process and isolation of the brain tumor stem cell $\left(\mathrm{CD} 133^{+}\right)$

\section{Acknowledgements}

This work was financed by Instituto Israelita de Ensino e Pesquisa Albert Einstein (IIEP/278-07). We are grateful to Laboratório de Microscopia Eletrônica, Departamento de Biologia - UNESP de Rio Claro, SP - Brasil and Antonio T. Yabuki and Monika Iamonte for the technical support.

\section{References}

Beier, D. et al. (2007). CD133+ and CD133- glioblastoma-derived cancer stem cells show differential growth characteristics and molecular profiles. Cancer Res., Vol. 67, N.9, pp. 4010-4015.

Brandes, A.A. et al. (2008). Glioblastoma in adults Critical. Reviews in Oncology/Hematology, Vol.67, pp. 139-152.

Central Brain Tumor Registry of the US. http://www.cbtrus.org/reports//20072008/2007report.pdf

Cheng, F.Y. et al. (2005). Characterization of aqueous dispersions of Fe3O4 nanoparticles and their biomedical applications. Biomaterials, Vol.26, pp. 729-738. 
Daldrup-Link, H.E. et al. (2003). Targeting of hematopoietic progenitor cells with MR contrast agents. Radiology, Vol.228, N.3, pp. 760-767.

Hartig, W., et al. (2003). Electron microscopic analysis of nanoparticles, delivering thioflavin-T after intrahippocampal injection in mouse: implications for targeting $\beta$ amyloid in Alzheimer`s disease. Neuroscience Letters, Vol.338, pp.174-176.

Jackson, H. et al. (2007). Quantum dots are phagocytized by macrophages and colocalize with experimental gliomas. Neurosurgery, Vol.60, N.3, pp. 524-529.

Jordan A., et al. (1997). Cellular uptake of magnetic fluid particles and their effects in AC magnetic fields on human adenocarcinoma cells in vitro. Int. J. Hypertherm., Vol. 13, pp. 587-605.

Jordan A. et al. (1999). Endocytosis of dextran and silan-coated magnetite nanoparticles and the effect of intracellular hyperthermia on human mammary carcinoma cells in vitro. J Magn. Magn. Mater, Vol.194, pp. 185-96.

Kumar, C.S.S.R. (2007). Nanomaterials for Cancer Diagnosis (WILEY-VCH), Weinheim.

Lee, J.H. et al. (2007). Artificially engineered magnetic nanoparticles for ultra-sensitive molecular imaging. Nature Medicine, Vol. 13, N.1, pp. 95-99.

Liu, M. et al. (2006). Fox M1B is overexpressed in human glioblstomas and critically regulates the tumorigenicity of glioma cells. Clin. Cancer Res. Vol. 66, N.7, pp. 35933602.

Louis, D.N. et al. (2007). The 2007 WHO classification of tumours of the central nervous system. Acta Neuropathol.,Vol. pp. 114:197.

Margaritescu, C. et al. (2008). Endoglin (CD105) and microvessel density in oral squamous cell carcinoma. Rom J Morphol Embryol. Vol.49, N.3, pp. 321-326.

Miraglia, S. et al. (1997). A novel five-transmembrane hematopoietic stem cell antigen: isolation, characterization, and molecular cloning. Blood. Vol. 90, pp. 5013-521.

Nisman, R. et al. (2004). Application of quantum dots as probes for correlative fluorescence, conventional, and energy-filtered transmission electron microscopy. Journal of Histochemistry \& Cytochemistry, Vol.52, N.1, pp. 13-18.

Reddy, G. R. et al. (2006). Vascular Targeted Nanoparticles for imaging and treatment of brain tumors. Clin Cancer Res., Vol. 12, N.22, pp. 6677-6686.

Rudzki, Z. \& Jothy S. (1997). CD44 and the adhesion of neoplastic cells. Mol. Pathol., Vol.50, pp. :57-71.

Simberg, D. et al. (2007). Biomimetic amplification of nanoparticle homing to tumors. PNAS, Vol. 104, N.03, pp.932-936.

Singh, S.K. et al. (2003). Identification of a cancer stem cell in human brain tumors. Cancer Res., Vol. 63, pp. 5821-5828.

Singh, S.K. et al. (2004). Identification of human brain tumour initiating cells. Nature, Vol.432, pp.396-401.

Sordi, V. et al. (2005). Bone marroe mesenchymal stem cell express a restricted set of sunctionally active chemokine receptors capacle of promoting migration to pancreatic islets. Blood, Vol.106, N.2, pp. 419-427.

Tartaj, P. et al. (2003). Preparation, structural and magnetic characterization of synthetic anti ferromagnetic (SAF) nanoparticles. Phys. D. Appl. Phy., Vol.36, pp. R182.

Tso, C. et al. (2006). Primary glioblastomas express mesenchymal stem-like properties. Mol. Cancer Res., Vol.4, N.9, pp. 607-619. 
Voltairas, P. A. et al. (2002). Hydrodynamics of magnetic drug targeting. J. Bioech.,Vol. 35, pp. 813-821.

Voura, E.B. et al. (2004). Tracking metastatic tumor cell extravasation with quantum dot nanocrystals and fluorescence emission-scanning microscopy. Nature Medicine, Vol.10, N.9, pp. 993-998.

Wen, P.Y. \& Kesari, S. (2008). Malignant gliomas in adults. N. Engl. J. Med., pp.359:492.

Zeppernick, F. et al. (2008). Stem cell marker CD133 affects clinical outcome in gliomas patients. Clin. Cancer Res., Vol.14, N.1, pp. 123-129. 


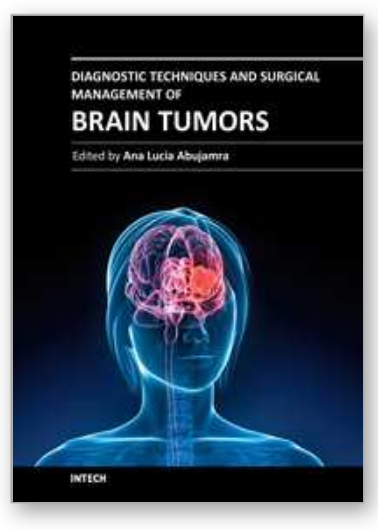

\author{
Diagnostic Techniques and Surgical Management of Brain Tumors \\ Edited by Dr. Ana Lucia Abujamra
}

ISBN 978-953-307-589-1

Hard cover, 544 pages

Publisher InTech

Published online 22, September, 2011

Published in print edition September, 2011

The focus of the book Diagnostic Techniques and Surgical Management of Brain Tumors is on describing the established and newly-arising techniques to diagnose central nervous system tumors, with a special focus on neuroimaging, followed by a discussion on the neurosurgical guidelines and techniques to manage and treat this disease. Each chapter in the Diagnostic Techniques and Surgical Management of Brain Tumors is authored by international experts with extensive experience in the areas covered.

\title{
How to reference
}

In order to correctly reference this scholarly work, feel free to copy and paste the following:

Lorena Favaro Pavon, Luciana Cavalheiro Marti, Tatiana Tais Sibov, Suzana M.F. Malheiros, Daniela Mara Oliveira, Maria Izabel Camargo-Mathias, Edson Amaro Junior ,Reynaldo Andre Brandt, Jorge Roberto Pagura, Marcos Augusto Stavale, Guilherme Carvalhal Ribas and Lionel Fernel Gamarra (2011). Immunolocalization of the Glioblastoma Cells by Nanoparticles Using Microscopy Tools, Diagnostic Techniques and Surgical Management of Brain Tumors, Dr. Ana Lucia Abujamra (Ed.), ISBN: 978-953-307-589-1, InTech, Available from: http://www.intechopen.com/books/diagnostic-techniques-and-surgical-management-of-braintumors/immunolocalization-of-the-glioblastoma-cells-by-nanoparticles-using-microscopy-tools

\section{INTECH}

open science | open minds

\section{InTech Europe}

University Campus STeP Ri

Slavka Krautzeka 83/A

51000 Rijeka, Croatia

Phone: +385 (51) 770447

Fax: +385 (51) 686166

www.intechopen.com

\section{InTech China}

Unit 405, Office Block, Hotel Equatorial Shanghai

No.65, Yan An Road (West), Shanghai, 200040, China

中国上海市延安西路65号上海国际贵都大饭店办公楼405单元

Phone: +86-21-62489820

Fax: +86-21-62489821 
(C) 2011 The Author(s). Licensee IntechOpen. This chapter is distributed under the terms of the Creative Commons Attribution-NonCommercialShareAlike-3.0 License, which permits use, distribution and reproduction for non-commercial purposes, provided the original is properly cited and derivative works building on this content are distributed under the same license. 